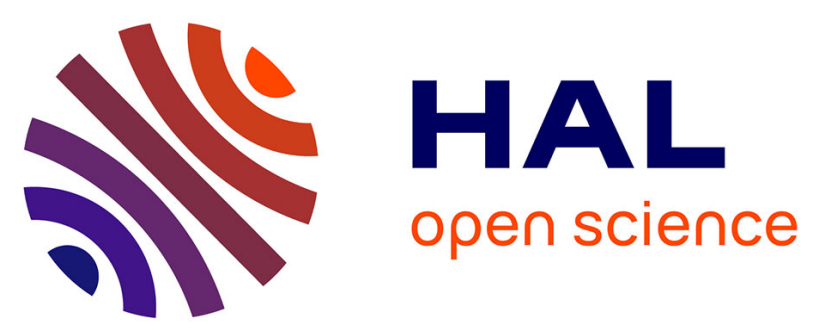

\title{
LES RAISONS DE L'ATTRIBUTION D'UNE FONCTION DE RÈGLEMENT DES DIFFÉRENDS AUX AUTORITÉS DE RÉGULATION : UNE COMPARAISON FRANCE - ROYAUME-UNI
}

Thomas Perroud

\section{To cite this version:}

Thomas Perroud. LES RAISONS DE L'ATTRIBUTION D'UNE FONCTION DE RÈGLEMENT DES DIFFÉRENDS AUX AUTORITÉS DE RÉGULATION : UNE COMPARAISON FRANCE ROYAUME-UNI. Droit et Société, 2016, Droit et société, 2/2016 (93), pp.317-336. hal-01448572

HAL Id: hal-01448572

https://hal.science/hal-01448572

Submitted on 30 Jan 2017

HAL is a multi-disciplinary open access archive for the deposit and dissemination of scientific research documents, whether they are published or not. The documents may come from teaching and research institutions in France or abroad, or from public or private research centers.
L'archive ouverte pluridisciplinaire HAL, est destinée au dépôt et à la diffusion de documents scientifiques de niveau recherche, publiés ou non, émanant des établissements d'enseignement et de recherche français ou étrangers, des laboratoires publics ou privés. 


\section{LES RAISONS DE L'ATTRIBUTION D'UNE FONCTION DE RĖGLEMENT DES DIFFÉRENDS AUX AUTORITÉS DE RÉGULATION : UNE COMPARAISON FRANCE - ROYAUME-UNI Thomas Perroud}

Editions juridiques associées | « Droit et société »

2016/2 Nº 93 | pages 317 à 336

ISSN 0769-3362

ISBN 9782275029016

Article disponible en ligne à l'adresse :

http://www.cairn.info/revue-droit-et-societe-2016-2-page-317.htm

\section{Pour citer cet article :}

Thomas Perroud, «Les raisons de l'attribution d'une fonction de règlement des différends aux autorités de régulation : une comparaison France - Royaume-Uni », Droit et société 2016/2 (N²3), p. 317-336.

Distribution électronique Cairn.info pour Editions juridiques associées.

(C) Editions juridiques associées. Tous droits réservés pour tous pays.

La reproduction ou représentation de cet article, notamment par photocopie, n'est autorisée que dans les limites des conditions générales d'utilisation du site ou, le cas échéant, des conditions générales de la licence souscrite par votre établissement. Toute autre reproduction ou représentation, en tout ou partie, sous quelque forme et de quelque manière que ce soit, est interdite sauf accord préalable et écrit de l'éditeur, en dehors des cas prévus par la législation en vigueur en France. Il est précisé que son stockage dans une base de données est également interdit. 


\title{
Les raisons de l'attribution d'une fonction de règlement des différends aux autorités de régulation: une comparaison France - Royaume-Uni
}

\author{
Thomas Perroud
}

Aix-Marseille Université, Faculté de Droit et de Science politique, 3 Avenue Robert Schuman, F-13628 Aix en Provence Cedex

<thomas.perroud@univ-amu.fr>

Résumé

En France et au Royaume-Uni, une fonction contentieuse a été confiée à des autorités indépendantes. Si leur pouvoir de sanction a beaucoup été étudié, il n'en va pas de même de leur pouvoir de règlement des litiges d'accès partagé à un réseau. Si l'analyse comparée des raisons de l'attribution d'une telle fonction à des régulateurs indépendants met en lumière une divergence d'approche historique de la question au Royaume-Uni et en France (approche liée aux théories économiques dans le premier cas, souci plus concret d'équité concurrentielle dans le second), une convergence est toutefois mise en évidence dans la mesure où, dans les deux cas, le phénomène prend sa source dans une cause structurelle, résidant dans des insuffisances du droit privé.

Accès au réseau - Droit de la concurrence - Droit privé des biens et des contrats - Fonction contentieuse - Règlement des litiges.

Summary The Judicial Function of Regulatory Agencies in France and the United Kingdom

In both countries powers were conferred upon regulatory agencies, namely the power to settle disputes on access to the networks. The aim of the article is to understand the rationale of such powers. If we try to understand the historical genesis of these powers, we may unearth some differences between the two countries. In France, Parliament want to foster an economic system based on fairness and therefore puts in place disputes resolution mechanisms in order to provide to the weakest party a swift and effective remedy. In the UK on the other hand, only the influence of economic theories has justified such an evolution. But, if we try to focus on more structural elements, it appears that, in both countries, it is the shortcomings of private law that explain the evolution.

Dispute Resolution - Competition Law - Judicial Powers - Network Access Private law. 
La fonction contentieuse des autorités de régulation en France et au RoyaumeUni est double : il s'agit, d'une part, d'un pouvoir de sanction et, d'autre part, d'un pouvoir de règlement des différends des litiges privés relatifs à l'accès au réseau ${ }^{1}$. La première ayant été déjà bien étudiée en France par la doctrine, nous nous concentrerons sur la seconde, qui a fait l'objet de moins d'analyses. La présente contribution cherche à retracer les raisons qui ont poussé les législateurs nationaux à conférer un tel pouvoir à ces agences. La fonction de règlement des différends occupe aujourd'hui une place importante dans les pouvoirs des régulateurs sectoriels et même, en France, au-delà, puisque ce pouvoir est présent dans le domaine du cinéma, de l'audiovisuel (Conseil supérieur de l'audiovisuel, CSA), de la propriété intellectuelle (Haute Autorité pour la diffusion des œuvres et la protection des droits sur Internet, HADOPI) et de la distribution de la presse (Autorité de régulation de la distribution de la presse). Ce pouvoir a pour objet de forcer le propriétaire d'un réseau d'ouvrir celui-ci à un concurrent afin qu'il puisse exercer son activité.

Dans notre thèse, nous avons défini cette fonction de règlement des différends comme un procédé de réglementation qui a pour but de résoudre un différend relatif à l'accès par l'émission d'une injonction ${ }^{2}$. Nous avons identifié deux grands types de causes permettant de rendre compte du développement de ce pouvoir. Nous voudrions exposer la naissance et le développement de ces pouvoirs en recourant à deux grilles d'analyse : l'une, historique, s'attachera à suivre pas à pas les législateurs nationaux en essayant de comprendre à chaque fois pourquoi ils ont choisi d'accorder de tels pouvoirs à ces agences. On verra à cet égard une différence entre la France et le Royaume-Uni (I). L'autre démarche consistera à comprendre comment, de manière structurelle, l'attribution de ces pouvoirs s'explique par les insuffisances du droit privé, et comment, sur ce point, les deux systèmes juridiques sont au contraire en harmonie (II).

\section{La divergence de fondement de la fonction de règlement des différends}

Il est très difficile de comprendre comment ce pouvoir est né. Rien dans les travaux préparatoires des différentes lois n'explique sa mise en place. Il faut donc procéder autrement pour comprendre comment l'idée de cette fonction administrative a pu naître. Dans le monde anglo-saxon, l'émergence de la fonction de règlement des différends ne peut se comprendre que dans le cadre des progrès réalisés par la théorie économique (l.1). En France, la fonction de règlement des différends s'explique à l'origine par un souci d'équité dans les relations concurrentielles (I.2). Enfin, nous tenterons de tracer les frontières actuelles du pouvoir de règlement des différends au Royaume-Uni et en France (I.3).

1. Cette contribution est issue des résultats de notre thèse : Thomas PERROUD, La fonction contentieuse des autorités de régulation en France et au Royaume-Uni, Paris: Dalloz, coll. "Nouvelle Bibliothèque de Thèses", 127, 2013.

2. Ibid., p. 376 et suiv. 


\section{I.1. Le fondement économique de la fonction de règlement des différends dans le monde anglo-saxon}

L'histoire de cette nouvelle fonction est difficile à retracer avec précision. Force est néanmoins de constater que c'est aux États-Unis que les idées ont émergé, à la fois économiquement, puisque c'est dans la doctrine économique que la question de l'accès et de son pendant technique, l'interconnexion, ont été théorisées et juridiquement mises en œuvre par les tribunaux, les autorités de régulation et le Congrès. L'émergence de cette fonction s'explique par la prise de conscience progressive de la nécessité de trouver un moyen simple et efficace pour obliger les opérateurs historiques à ouvrir leur réseau. C'est une solution toute pragmatique à un problème récurrent que les juridictions se sont avérées incapables de résoudre de manière efficace.

Les États-Unis ont connu une évolution assez similaire à l'Europe dans la réglementation des services publics en réseau. Ce pays a eu certes moins recours à la gestion publique directe, sauf dans le domaine postal ${ }^{3}$, mais dans l'ensemble des secteurs le monopole - le plus souvent d'ailleurs local - était de mise.

L'évolution qui mène, aux États-Unis, de l'activité exercée en monopole sous la protection d'un droit exclusif, à la déréglementation totale dans certains cas, comme l'aviation, ou à la reconfiguration de l'intervention de l'autorité administrative, ne peut s'expliquer sans référence à l'évolution profonde de la pensée économique qui caractérise ces années ${ }^{4}$. Les économistes commencèrent à remettre en question les postulats économiques de la réglementation classique : tant la justification même de la réglementation (la théorie du monopole naturel) que ses effets ou ses buts (le bénéfice du consommateur), que ses deux outils majeurs (la restriction à l'entrée sur le marché par l'intermédiaire des droits exclusifs 5 , et la réglementation des prix). L'innovation de la politique américaine, qui naît dans le secteur des télécommunications et de l'énergie, résulte d'une double prise de conscience. D’une part, les économistes se rendent compte du hiatus entre le monopole véritablement naturel et le monopole juridique. D'autre part, ceux-ci vont concentrer le feu de leurs critiques sur les instruments juridiques d'encadrement des secteurs: l'autorisation administrative préalable qui ferme le marché et la police des prix. Ces deux changements traduisent un bouleversement de la conception même du marché.

3. Ce monopole dans le secteur postal est d'ailleurs à l'origine de l'engagement d'un des plus célèbres « libertarien » américain, Lysender Spooner, qui avait créé son entreprise de transport et de distribution de courrier, dont il se vit privé par le Congrès, qui vota une loi instituant un monopole étatique dans le domaine postal. Voir Lysender SPOONER, The Unconstitutionality of the Laws of Congress Prohibiting Private Mail, New York : Printing Establishment, 1944.

4. Voir l'affirmation très nette de l'influence de la pensée économique sur la réforme in Martha DERTHICK et Paul J. QuIRK, The Politics of Deregulation, Washington, D.C. : Brookings Institution, 1985, p. 36 ; Voir aussi Christopher David FosTER, Privatization, Public Ownership and the Regulation of Natural Monopoly, Oxford : Blackwell, 1992, p. 373.

5. L'École de Chicago, suivant en cela les travaux de Stigler, remet fortement en question la légitimité des barrières à l'entrée. 


\section{La remise en question de la notion de monopole naturel}

L'existence d'un monopole naturel ${ }^{6}$ est, dans certains domaines, remise en question. À la fin du XIX et au début du Xx $x^{e}$ siècle, la concurrence au niveau local dans les services publics en réseau s'est avérée incapable d'atteindre des résultats satisfaisants. La notion de monopole naturel fut alors avancée pour rendre compte de cet échec et justifier l'exercice de l'activité en monopole, c'est-à-dire en régime de droit exclusif. Le rôle de l'autorité publique, dans ce cadre, était de protéger l'opérateur historique ${ }^{7}$. Les études économiques vont tenter de montrer d'abord que bien souvent le monopole naturel, au sens économique du terme, ne recouvre pas l'ensemble du monopole effectif, au sens juridique du terme. En effet, les entreprises en monopole ont progressivement étendu leurs activités à des domaines connexes, comme la fabrication et la vente de téléphones pour l'opérateur de télécommunication. Ce secteur, la fabrication d'équipements de télécommunications, ne fait pas partie du monopole naturel. Ensuite, une concurrence a commencé à s'exercer entre différentes technologies : pour le transport, le train se voit concurrencé par la route; dans l'énergie, le gaz est, lui, menacé par de nouvelles énergies comme le pétrole. Au total, l'évolution des technologies rend la justification du monopole naturel inadaptée aux conditions présentes: dans bien des cas, une concurrence opère. Dans d'autres secteurs comme le transport routier ou l'aviation, les recherches montrent que ceux-ci ont été à tort assimilés à des monopoles naturels 8 . Ce sont ces secteurs qui subissent la première véritable déréglementation ${ }^{9}$, dans le sens qu'ils ne sont plus encadrés que par les règles du droit commun, c'est-à-dire du droit privé et du droit de la concurrence. Avec la remise en cause de la théorie du monopole naturel, on constate aussi une remise en question du pouvoir de police.

\section{La remise en cause de la police : le pouvoir d'autorisation et la police des prix}

L'intérêt des outils principaux de la réglementation à savoir l'attribution de droits par l'intermédiaire de l'autorisation administrative préalable est fortement remis en question. Il apparait que le système de l'autorisation préalable est le principal outil juridique qui fait l'objet des attaques des économistes, car il constitue une barrière légale à l'entrée sur le marché. L'histoire des différents secteurs

6. La littérature sur cette notion, même juridique, retient une définition économique qui repose sur la notion d'échec de marché. L'existence d'un monopole naturel est un cas classique, à côté de celle d'externalités et de biens communs, d'échec de marché. Elle est justifiée, car, dans ce cas, le monopole est plus bénéfique pour la société, le service étant produit à moindre coût, en raison de l'existence d'économie d'échelle. Le problème est qu'il s'agit d'une définition a posteriori: un monopole naturel existe lorsqu'une seule compagnie produit des biens et des services à un coût moindre que deux compagnies. Voir sur cette notion François LEVÊQUE, Économie de réglementation, Paris : La Découverte, coll. « Repères », 1998, spéc. p. 51.

7. Christopher David FosTer, Privatization, Public Ownership and the Regulation of Natural Monopoly, op. cit., p. XX; Dominique Custos, La Commission fédérale américaine des communications à l'heure de la régulation des autoroutes de l'information, Paris : L'Harmattan, coll. «Logiques juridiques », 1999, p. 333.

8. Les raisons qui ont incité à la réglementation du secteur aéronautique sont essentiellement le caractère destructeur de la concurrence dans ce secteur dans les années 1930 (voir Alfred Edward KAHN, «The Political Feasibility of Regulatory Reform: How Did we Do it? ", in LeRoy GrAYMER et Frederick THOMPSON (eds.), Reforming Social Regulation Alternative Public Policy Strategies, Los Angeles: Sage Publications, 1982, p. 247-263, spéc. p. 250).

9. Airline Deregulation Act (Pub. L. 95-504), du 24 octobre 1978. 
montre que, même en l'absence de droits exclusifs, les autorités furent très réticentes à accorder de nouvelles autorisations pour augmenter le nombre de concurrents. Dans le domaine aéronautique, le Civil Aeronautics Board (le régulateur du secteur aéronautique) n'accorda aucune autorisation supplémentaire. Les seize compagnies exerçant leur activité dans ce secteur en 1938 n'étaient même plus que dix dans les années 1970, en raison des mouvements de fusion qu'il connut.

L'autre instrument classique, la police des prix, subit, lui aussi, le feu de la critique. La réglementation des prix utilisait comme standard le taux de rendement qui devait être calculé par l'autorité administrative de manière à être juste et raisonnable. Des promoteurs de la tarification au coût marginal commencèrent à faire entendre leur voix et, parmi eux, le plus fervent en théorie et le plus influent en pratique fut Alfred E. Kahn ${ }^{10}$. Cette proposition revenait en réalité, pour l'autorité administrative, à jouer le même rôle que le marché puisqu'en théorie microéconomique le prix est équivalent au coût marginal ${ }^{11}$. La police des prix, pour être légitime, doit dès lors reproduire l'effet du marché.

\section{Le renouvellement de la conception du marché}

Mais ces critiques ne doivent pas masquer que le changement réel se situe à un autre niveau : la conception même du marché a changé. Friedrich Hayek a fortement contribué à ce mouvement en montrant qu'il ne fallait plus s'intéresser à la concurrence réelle, car la concurrence n'est pas un phénomène statique, c'est au contraire un phénomène dynamique, une procédure de découverte ${ }^{12}$. Autrement dit, ce n'est pas parce qu'un marché ne connaît pas actuellement un niveau de concurrence adéquat qu'il faut intervenir, le marché va de lui-même, dans le temps, rectifier le problème.

La prise en compte du temps dans la conception du marché se retrouve dans une autre théorie qui a joué un rôle crucial dans l'ouverture à la concurrence des industries en réseau: la théorie de la contestabilité (the theory of contestable markets) ${ }^{13}$. C'est cette théorie qui permet de comprendre les changements intervenus

10. Alfred Edward KAHN, The Economics of Regulation, Principles and Institutions, vol. I, Cambridge : MIT Press, 1988, p. 65.

11. Le coût marginal est défini comme le coût que supporte l'opérateur pour produire une unité supplémentaire. Pourquoi faut-il tarifer au coût marginal ? L'utilisation de cet indicateur s'explique par la nécessité de partir du point de vue du consommateur. Celui-ci doit faire face à un choix, acheter plus ou moins certains produits. Dans une économie caractérisée par la rareté, le choix du consommateur se traduira par une sélection d'opportunités, consommer davantage, ou au contraire, moins, tel ou tel produit. Si le prix reflète ce coût d'opportunité, les consommateurs opéreront un choix éclairé : leur satisfaction en écartant certains biens au profit d'autres sera alors optimale et surtout les ressources nécessairement limitées se dirigeront vers les produits qui rencontreront le suffrage le plus important de la part des consommateurs. En conséquence, si les tarifs ne reflètent pas le coût marginal, l'optimum n'est pas atteint, soit les consommateurs consommeront trop, ce qui se traduira par un gaspillage, soit ils ne consommeront pas suffisamment. Voir notamment Alfred Edward KAHN, The Economics of Regulation, Principles and Institutions, vol. I, op. cit., p. 66-67. Voir aussi Paul A. SAMUELSON et William D. NoRdHAUS, Économie, Paris : Economica, $16^{\mathrm{e}}$ éd., spéc. p. 138-140 (en concurrence pure et parfaite le prix est égal au coût marginal).

12. Friedrich A. HAYEK, "Competition as a Discovery Procedure", The Quarterly Journal of Austrian Economics, 5 (3), p. 9-23.

13. Ejan MaCKAaY et Stéphane Rousseau, Analyse économique du droit, Paris : Dalloz, coll. « Méthodes du droit ", $2^{\mathrm{e}}$ éd., 2008, spéc. p. 104. 
dans les différents secteurs et, surtout, la mise en place, de la fonction de règlement des différends devant l'autorité administrative. Elle a eu une influence considérable sur la politique mise en place par les autorités sectorielles. Le modèle de la contestabilité, qui s'inscrit et ne peut se comprendre que dans le cadre plus large de la réflexion sur la redéfinition de la notion de barrière à l'entrée d'un marché ${ }^{14}$, se présente comme un moyen de changer de paradigme face au modèle de la concurrence pure et parfaite. Ce changement de paradigme permet de penser autrement la politique publique, les modalités de l'intervention de l'État. Alors que dans le modèle de concurrence pure et parfaite, tout écart, tout échec du marché peut justifier l'intervention de l'État et éventuellement l'existence d'un monopole légal, les promoteurs de la contestabilité (William J. Baumol, John C. Panzar et Robert D. Willig) vont montrer que le simple fait qu'un marché soit contestable suffit à discipliner l'entreprise présente sur celui-ci ${ }^{15}$. Ce changement de référence bouleverse alors la manière dont la politique publique doit être pensée. L'expression des conséquences politiques de ce bouleversement théorique revint à Elizabeth E. Bailey qui travailla au Civil Aeronautics Board ${ }^{16}$. Si la contestabilité suffit à discipliner correctement l'opérateur historique, car la concurrence pour le marché a un effet disciplinant, celle-ci rend l'intervention publique inutile, ou plutôt, cette intervention doit être repensée, non plus pour protéger les consommateurs puisque cette protection est désormais inutile, car assurée par la peur des nouveaux entrants potentiels, mais, au contraire, pour promouvoir la contestabilité. Pour ce qui nous intéresse, la théorie de la contestabilité a une influence directe sur le droit.

Le standard n'est donc plus la concurrence pure et parfaite, mais un nouveau modèle : le marché parfaitement contestable, qui est un marché ouvert à la concurrence et dans lequel les nouveaux entrants ne font face à aucun désavantage par rapport aux entreprises présentes et duquel ils peuvent sortir sans coûts excessifs. L'intervention publique doit donc être orientée, dans ce cadre, vers l'augmentation de la contestabilité des marchés, ce qui a deux conséquences.

\footnotetext{
14. L'École de Chicago remet complètement en question l'importance des barrières à l'entrée sur le marché, mises en avant par l'école structuraliste. Cette notion est considérablement réduite pour ne désigner que les coûts de production supplémentaires supportés par l'entreprise qui veut entrer sur le marché et que ne supportent pas les entreprises déjà présentes. Cette redéfinition fait perdre à la notion toute portée, car tous les investissements que l'entreprise devra réaliser le sont déjà par les acteurs présents, si bien que ceux-ci ne disposent pas, en réalité et contrairement à ce que pensait la théorie classique, d'un avantage particulier qui nuirait à l'entrée. Voir Marc Allen EISNER, Antitrust and the Triumph of Economics: Institutions, Expertise, and Policy Change, Chapel Hill : University of North Carolina Press, 1991, p. 103.

15. L'ensemble de la pensée de ces auteurs et leurs différents articles écrits sur le sujet pendant les années 1970 est contenu dans William J. BAUMOL, John C. PANZAR et Robert D. WILLIG, Contestable Markets and the Theory of Industry Structure (avec les contributions de Elizabeth E. BAILEY, Dietrich FisCHER, Herman C. QuiRmBACH), New York: Harcourt Brace Jovanovich, 1982. La réflexion sur ce sujet a aussi été initiée par Harold DEMSETZ, «Why Regulate Utilities », Journal of Law \& Economics, 11, apr. 1968, p. 55-65.

16. Elle exprime et développe les conséquences politiques de la théorie de la contestabilité à la fois dans un article: "Contestability and the Design of Regulatory and Antitrust Policy", The American Economic Review, 71 (2), Papers and Proceedings of the Ninety-Third Annual Meeting of the American Economic Association (May, 1981), p. 178-183, et dans l'ouvrage de William J. BAUMOL, John C. PANZAR et Robert D. WILLIG, Contestable Markets and the Theory of Industry Structure, op. cit.
} 
D'une part, il faut supprimer ou diminuer les règles juridiques qui réduisent la contestabilité du marché en érigeant des barrières à l'entrée sur ce marché. La politique d'autorisation préalable doit donc être repensée. La contestabilité a aussi une conséquence sur la police des prix puisque, pour que le marché soit contesté, il est nécessaire que les nouveaux arrivants puissent pratiquer des prix plus faibles.

D'autre part, les politiques publiques doivent désormais tendre à augmenter la contestabilité. La barrière à l'entrée sur le marché la plus dirimante réside dans les coûts de sortie de celui-ci. Si ces coûts sont irrécupérables, c'est-à-dire si ce sont des coûts que le nouveau compétiteur devra supporter pour entrer sur le marché sans pouvoir les récupérer s'il échoue devant l'opérateur historique, cela peut constituer un obstacle définitif à la contestabilité du marché. Dans ce cas, qui nous intéresse particulièrement puisque, dans l'ensemble des industries en réseau, il y a des coûts irrécupérables importants, la politique doit être orientée spécifiquement vers l'abolition ou l'aménagement de ceux-ci.

Elizabeth E. Bailey explique ainsi que les moyens pour diminuer les barrières à l'entrée, liées à l'acquisition d'infrastructures coûteuses, doivent être orientés vers la mise en place de mécanismes qui rendent bénin l'exercice du pouvoir associé à ces infrastructures 17 . Elle propose donc soit la nationalisation de ces infrastructures sur le modèle des autoroutes et des routes qui sont la propriété de la collectivité, soit la mise en place d'un consortium qui deviendrait propriétaire de ces infrastructures. Les règles doivent favoriser l'accès de manière non discriminatoire et permettre le transfert, la location ou l'utilisation commune de la manière la plus aisée ${ }^{18 .}$.

On comprend bien à quel point cette théorie et le changement de paradigme qu'elle emporte sont à l'origine de la fonction de règlement des différends, d'abord en ce qu'elle met comme point focal, essentiel, de la politique, non plus la protection des usagers comme auparavant, mais l'accès au marché pour rendre celui-ci contestable. Or, pour qu'un marché tel que celui des services publics en réseau soit contestable, il faut bien que les concurrents puissent avoir accès à celui-ci. Cette exigence permet d'expliquer pourquoi la loi est intervenue pour accorder aux concurrents un droit d'accès aux réseaux des opérateurs historiques aussi bien dans le domaine des télécommunications, que de l'énergie ou des postes.

Au Royaume-Uni, dans les années 1990, la question de l'accès à ce réseau va naturellement poser celle des pouvoirs de l'autorité administrative pour forcer l'opérateur propriétaire à ouvrir celui-ci. La consultation menée par le gouvernement britannique de l'époque pour réfléchir aux évolutions nécessaires reconnaît ainsi la nécessité de pouvoir s'en remettre au Directeur général des télécommunications au cas où l'opérateur renâclerait à contracter ${ }^{19}$. La lecture des rapports montre bien que la fonction de règlement des différends est perçue comme une garantie par les

17. Elizabeth E. BAILEY, « Contestability and the Design of Regulatory and Antitrust Policy », art. cité, p. 179.

18. Ibid.

19. OFTEL, « Duopoly Review », 1990, p. 54, n 9.21 . 
acteurs de ce marché 20 . Au début des années 1990, la physionomie de la fonction de règlement des différends apparaît plus clairement et sera progressivement étendue à l'ensemble des secteurs en réseau. En France, en revanche, la consécration de cette fonction ne se fera pas dans les secteurs en réseau, mais dans celui du cinéma, montrant, dans ce cas, que c'est un souci d'équité dans les relations concurrentielles qui motive davantage l'attribution de ce pouvoir à une autorité administrative: la création du Médiateur du cinéma est ainsi emblématique.

\section{I.2. La naissance du règlement des différends en France : le souci de l'équité}

La première manifestation du règlement des différends en France concerne le secteur du cinéma, où, pour la première fois, une autorité administrative indépendante est dotée d'un pouvoir d'enjoindre des parties à contracter. La seconde manifestation, elle, sera plus discrète : il s'agira de l'émergence de ce pouvoir dans le secteur des télécommunications.

\section{Le pouvoir de règlement des différends du Médiateur du cinéma}

La première autorité administrative dotée d'un pouvoir de règlement des différends en France pour une question d'accès est le Médiateur du cinéma. Ce pouvoir a d'ailleurs été confirmé par l'ordonnance du 5 novembre 2009 modifiant le Code du cinéma et de l'image animée ${ }^{21}$. Le Médiateur du cinéma, créé par la loi du 29 juillet 1982 sur la communication audiovisuelle 22 est à l'époque, et encore dans une large mesure aujourd'hui, une "institution originale qui n'existe dans aucun autre pays ni dans aucun autre secteur de l'activité économique ou culturelle en France » 23 . Cette institution dispose, depuis l'origine, d'un pouvoir de conciliation des « litiges relatifs à la diffusion en salle des œuvres cinématographiques et qui ont pour origine une situation de monopole de fait, une position dominante ou tout autre situation ayant pour objet ou pouvant avoir pour effet de restreindre ou de fausser le jeu de la concurrence et révélant l'existence d'obstacles à la plus large diffusion des œuvres cinématographiques conforme à l'intérêt général ». Le Médiateur disposait, dès la loi de 1982, d'un pouvoir contraignant qui rend son office très semblable à celui des autres autorités entrant dans le champ de cette recherche et qui justifie dès lors son étude. C’est aussi l'avis de Michèle Guillaume-Hofnung pour laquelle la faculté de prononcer une injonction est "radicalement incompatible avec l'absence de pouvoir qui caractérise le médiateur »24. De même, pour

20. Department of Trade And Industry, Competition and Choice: Telecommunications Policy for the 1990s, 1990-1991, Cm 1461, spéc. $\mathrm{n}^{\circ} 7.15$.

21. Article 3 de l'ordonnance ${ }^{\circ}$ 2009-1358 du 5 novembre 2009 modifiant le Code du cinéma et de l'image animée, codifié à l'article L. 213-4 du Code du cinéma et de l'image animée.

22. Article 92 al. 2 de la loi n $82-652$ du 29 juillet 1982 sur la communication audiovisuelle. Son statut est fixé par le décret n 83-86 du 9 février 1983 portant application des dispositions de l'article 92 de la loi n 82 652 du 29 juillet 1982 sur la communication audiovisuelle et relatif au médiateur du cinéma modifié par le décret $\mathrm{n}^{\circ}$ 91-1129 du 25 octobre 1991.

23. Voir les propos du ministre de la Culture sur le projet de loi, JORF, Débats, Assemblée nationale, compte rendu intégral, $2^{\mathrm{e}}$ séance du samedi 15 mai 1982, p. 2253.

24. Michèle Guillaume-Hofnung, "La médiation", L'actualité juridique. Droit administratif (AJDA), 1997, p. 30 . 
Laurent Richer, le Médiateur du cinéma n'est pas un médiateur, mais bien plutôt un conciliateur 25. Gérard Cornu définit quant à lui le médiateur comme «celui auquel les parties à un conflit demandent de proposer la solution de leur différend, sans être cependant investi du pouvoir juridictionnel de l'imposer » 26 . On s'aperçoit alors que le Médiateur du cinéma en question ne répond pas à cette définition puisqu'il détient précisément un pouvoir d'injonction. S'il dispose bien d'une mission de conciliation ou de médiation, dans le sens où sa mission première est d'aider les parties à parvenir à un accord, à celle-ci se superpose un pouvoir de règlement des différends, tel qu'il est mis en œuvre devant les autres autorités de régulation.

Comment expliquer la création, en 1982, de cette première institution de règlement des différends ? Le chemin qui mène à la création du Médiateur et à la mise en place d'un pouvoir contraignant est assez tortueux. Dès la fin des années 1970, une série de batailles judiciaires entre exploitants de salles et distributeurs amène des exploitants de salles indépendants à saisir la Commission de la concurrence. Ces derniers voulaient dénoncer certaines pratiques d'entente et d'abus de position dominante mises en œuvre par ces distributeurs au détriment des petits exploitants. Dans un avis du 28 juin 1979, la Commission stigmatisa ces comportements et recommanda des mesures au regard du droit de la concurrence et, surtout, évoqua la mise en place d'un « code de bonne conduite dont l'élaboration est actuellement poursuivie avec le concours des parties concernées et des administrations intéressées » 27 . Cette idée sera reprise dans la décision que le ministre rendra sur l'avis de la Commission de la concurrence sur les pratiques incriminées ; celui-ci invite les destinataires de cette décision «à participer à l'élaboration d'un code de bonne conduite en vue de normaliser les rapports entre distributeurs et exploitants de films cinématographiques » 28 .

Comment en est-on arrivé d'un code de bonne conduite à l'établissement d'une procédure de conciliation dont l'issue peut être, le cas échéant, contraignante? La volonté est de remédier à la situation de position dominante de certains exploitants 29 et, là encore, la Commission de la concurrence est au centre de ce processus. Celle-ci rend en effet un avis, le 25 mars $1982^{30}$, sur le projet de loi créant le Médiateur qui manifeste une nette évolution de la part de cette instance sur l'ampleur des pouvoirs dont devra jouir la future autorité. La proposition d'un code de bonne conduite semble désormais insuffisante, car la Commission plaide désormais pour l'institution

25. Laurent RICHER, «Arbitrage et conciliation », Répertoire de contentieux administratif, Dalloz, mars 2001, spéc. $\mathrm{n}^{\circ} 18$.

26. Gérard CoRnu, Vocabulaire juridique, Paris : PUF, 6 éd., 2004.

27. Avis du 28 juin 1979 de la Commission de la concurrence concernant la diffusion des films cinématographiques (BOCCRF, Bulletin officiel des services des prix, 21 du samedi 20 octobre 1979, p. 270).

28. Décision $\mathrm{n}^{\circ} 79-20$ du ministre de l'Économie du 29 octobre 1979 concernant la diffusion des films cinématographiques, BOCCRF, Bulletin officiel des services des prix, 22, mardi 30 octobre 1979, p. 283. Voir aussi décision nº 79-20 du ministre de l'Économie du 11 octobre 1979.

29. Trois exploitants de salles, le groupement d'intérêt économique Gaumont-Pathé, UGC et Parafrance disposaient d'une position dominante très nette sur le marché.

30. Avis du 25 mars 1982 rendu par la Commission de la concurrence sur un projet de loi concernant la diffusion des œuvres cinématographiques, BOCCRF, Bulletin officiel des services des prix, 4, mercredi 23 février 1983, p. 67. 
d'« une procédure accélérée de règlement des litiges relatifs à la diffusion des films ». Elle estime même : "L'autorité et l'efficacité du médiateur seraient renforcées s'il pouvait soit régler les désaccords par voie de conciliation, soit, à défaut de conciliation, émettre des recommandations. Ces recommandations pourraient être rendues publiques ${ }^{31}$.»

Les travaux parlementaires permettent aussi de comprendre les facteurs qui furent pris en compte dans le choix d'une autorité administrative ad hoc. Les parlementaires relèvent ainsi, qu'en pratique, le recours au droit de la concurrence est « inefficace » 32 , l'utilisation de l'infraction de refus de vente s'avérant inadaptée au contexte des petits exploitants qui avaient besoin d'une solution simple et rapide leur permettant de répondre à leur problème pratique immédiat : obtenir une copie du film. Mais comment en arrive-t-on à la mise en place d'un pouvoir contraignant, d'un pouvoir d'injonction?

Ce pouvoir, qui donne son caractère contraignant à la procédure devant le Médiateur, fut d'abord suggéré par la Mission de réflexion et de proposition sur le cinéma présidée par Jean-Denis Bredin ${ }^{33}$. La mission s'est d'abord concentrée sur les règles de fond qui devront régir le secteur. Ces règles reposent sur un principe : le distributeur conserve la responsabilité juridique de la commercialisation du film, il garde donc le pouvoir de refuser un film à un exploitant de salle. Ce principe est toutefois atténué dans sa portée par une règle servant à contrôler les motifs du refus de fournir un film, motifs qui ne sauraient être abusifs. Le rapport ajoute - ce qui semble être le fondement de l'institution d'un médiateur doté d'un pouvoir contraignant :

Sans doute, ces règles exigent-elles une appréciation délicate des caractéristiques respectives des films et des salles. Au fur et à mesure que l'analyse s'affine s'esquisse la nécessité d'une autorité extérieure. Aussi vraie que toute politique de la concurrence, appliquée à la trame complexe du tissu économique, s'exerce fatalement au moyen d'une jurisprudence, une instance indépendante s'impose pour adapter les principes qui viennent d'être énoncés à la diversité des situations particulières 34 .

Le pouvoir d'injonction est donc conçu comme le facteur d'individualisation des règles gouvernant le secteur. Ce pouvoir a ensuite été introduit par amendement en cours de discussion à l'Assemblée nationale. Il vise à "renforcer l'autorité du médiateur ${ }^{35}$. L'esprit de cette réforme est explicité par Roland Dumas dans les débats :

Il s'agit, en réalité, de faire en sorte que le médiateur puisse exercer son autorité avec le maximum d'efficacité. Le projet du gouvernement ne lui donne qu'un pouvoir de conciliation. Nous avons pensé qu'on pouvait aller au-delà en fixant comme limite, dans la loi, que le médiateur n'aurait pas de pouvoir juridictionnel, car nous ne voulons pas instituer une juridiction particulière. Par contre, le médiateur pourrait, après une tentative de conciliation, et en cas d'échec de celle-ci, prononcer une injonction et éventuellement la rendre publique. Il pourrait de la même façon saisir la commission de la concurrence pour des faits sur lesquels celle-ci serait compétente et - j'en

31. Avis du 25 mars 1982, op. cit., § 3 .

32. Voir les propos d'Alain Madelin, JORF, Débats, Assemblée nationale, $2^{\mathrm{e}}$ séance du 15 mai 1982, p. 2263.

33. Rapport de la Mission de réflexion et de propositions sur le cinéma, remis à M. le ministre de la Culture le 3 novembre 1981, par Jean-Denis Bredin, Paris, ministère de la Culture, 1981, p. 59.

34. Ibid.

35. Ministre de la Culture, JORF, Débats, Assemblée nationale, $2^{\mathrm{e}}$ séance du 15 mai 1982, p. 2264. 
arrive à la différence entre le texte du gouvernement et le mien - il pourrait également, lorsque cela lui paraîtrait nécessaire, saisir le ministère public pour un fait délictueux. Le pouvoir d'injonction, en réalité, n'est pas un pouvoir de décision juridictionnelle. C'est un degré supplémentaire par rapport à la recommandation. Je précise au passage que l'injonction est une notion assez fréquente dans le droit français. Nous la trouvons aussi bien dans le droit privé que dans le droit administratif. Ce n'est donc pas une innovation. D'autre part, la légère sanction qui accompagnerait l'injonction serait la manifestation publique du médiateur lorsqu'il ferait communication de son injonction. Il apparaît donc, dans ces conditions, qu'on va jusqu'à l'extrême limite du pouvoir que l'on peut donner à ce médiateur hormis, je répète, le pouvoir juridictionnel ${ }^{36}$.

Les pouvoirs du Médiateur du cinéma sont donc une création très progressive, pragmatique. Dans le secteur des télécommunications, l'émergence d'un tel pouvoir sera encore plus discrète.

\section{Le pouvoir de règlement des différends dans le secteur des télécommunications}

À la suite de cette expérience, c'est le secteur des télécommunications qui sera concerné par l'attribution d'un pouvoir administratif de règlement des différends. Ce pouvoir est à cette époque très discret puisqu'il est contenu dans le cahier des charges de l'opérateur historique France Télécom. L'article 11 de ce document 37 confère ainsi au ministre chargé des Postes et Télécommunications un pouvoir de règlement des différends 38 .

Il y a peu d'éléments sur la genèse de cette fonction. L'avis du 5 décembre 1990 de la Commission supérieure du service public des postes et télécommunications ne fait que prendre acte de cette nouvelle fonction en affirmant qu'elle est cohérente avec le « rôle de régulateur du secteur » qui échoit au ministre ${ }^{39}$. De même, le rapport du 31 juillet 1989 sur l'avenir du service public de la poste et des télécommunications se borne à constater :

En cas de litige, les différentes parties peuvent toujours saisir l'autorité de réglementation qui a la charge de faire respecter les règles, puis faire appel auprès des tribunaux ou du Conseil de la concurrence. Il serait souhaitable, avant d'entreprendre ces procédures lourdes, de pouvoir saisir une organisation chargée d'examiner les doléances et de donner un avis pour rapprocher les parties ou de proposer une solution à l'amiable ${ }^{40}$.

36. Assemblée nationale, compte rendu intégral des débats, $2^{\mathrm{e}}$ séance du 15 mai 1982, p. 2264.

37. Il est annexé au décret $n^{\circ}$ 90-1213 du 29 décembre 1990 relatif au cahier des charges de France Télécom.

38. Décret $n^{\circ}$ 90-1213 du 29 décembre 1990 relatif au cahier des charges de France Télécom et au Code des postes et télécommunications, JORF, 303, 30 décembre 1990, p. 16568. L'article 11 du cahier des charges dispose : «Ces droits d'accès sont négociés entre France Télécom et le demandeur et portés à la connaissance du ministre chargé des postes et télécommunications. En l'absence d'accord, chacune des deux parties peut saisir le ministre, qui peut alors fixer ces droits en tenant compte de la réalité des coûts. " Voir Élisabeth RoLIN, "Les règlements de différends devant l'Autorité de régulation des télécommunications ", in Marie-Anne Frison-Roche (dir.), Droit et économie de la régulation. 1, Les régulations économiques: légitimité et efficacité, Paris : Presses de Sciences Po, Dalloz, coll. «Thèmes et commentaires », 2004, p. 149-173, spéc. p. 151. Le ministre a rendu quatre décisions : voir Isabelle CIUPA, L'interconnexion dans les télécommunications : le concept d'ONP comme outil d'une régulation concurrentielle, Lille : ANRF, 1996, p. 287-288.

39. Avis en date du 5 décembre 1990 de la Commission supérieure du service public des postes et télécommunications (non publié), p. 43.

40. Rapport de synthèse remis par Hubert Prévot à Paul Quilès, ministre des Postes, des Télécommunications et de l'Espace, à l'issue du débat public sur l'avenir du service public des Postes et des Télécommunications, 31 juillet 1989, p. 79 . 
Les années 1980 et 1990 ont donc vu se développer la fonction de règlement des différends devant les autorités de régulation. Sa physionomie juridique est déjà fixée.

\section{I.3. La physionomie actuelle du pouvoir de règlement des différends au Royaume-Uni et en France}

L'identification de ces deux grands types de causes, la théorie économique et un souci d'équité, permet de comprendre pourquoi, aujourd'hui, il y a beaucoup plus d'autorités dotées d'un pouvoir de règlement des différends en France qu'au Royaume-Uni, où ce pouvoir est limité aux services publics en réseau. Dans le domaine des communications électroniques et des postes, il est confié à OFCOM, dans celui de l'énergie à OFGEM, pour le rail à l'Office of Rail Regulation et dans le domaine de l'eau à OFWAT ${ }^{41}$. En France, on retrouve un tel pouvoir dans les mêmes secteurs: l'ARCEP est chargé du règlement des différends dans les domaines des communications électroniques et des postes, la CRE dans celui de l'énergie et l'ARAFER dans le secteur ferroviaire ${ }^{42}$. Cependant, ce souci d'équité explique que cette fonction ait débordé ce cadre en France pour investir, en plus du cinéma, l'audiovisuel, la propriété intellectuelle et la distribution de la presse ${ }^{43}$. Le pouvoir de règlement des différends joue donc un autre rôle en France. Il marque l'intervention grandissante de l'Administration dans les relations commerciales privées pour résoudre des problèmes concrets de concurrence lorsque les sanctions ex post de l'Autorité de la concurrence ne seraient pas jugées suffisamment efficaces.

L'analyse historique nous a permis de mettre en évidence des motifs différents dans les deux pays. Le souci d'équité dans les relations concurrentielles qui justifierait de défendre certains acteurs comme les propriétaires indépendants de salles de cinéma en France, est absent au Royaume-Uni. Nous voudrions à présent mettre en évidence qu'une raison commune justifie d'attribuer, dans les deux pays, une telle fonction aux agences de régulation : les insuffisances du droit privé. Celui-ci ne permet pas, en effet, de répondre aux problèmes que posent les secteurs régulés, si bien qu'il a semblé indispensable de créer cette nouvelle fonction juridique.

\section{Une raison de fond commune : les insuffisances des droits privés anglais et français face à l'exigence d'accès}

Le droit privé apparaît inadapté pour répondre aux enjeux que présente le droit des secteurs régulés. La fonction de règlement des différends est, en effet, tout entière tournée vers le contrat, contrat de droit privé dans chaque pays. Certains caractères de la théorie générale des contrats de droit privé, en France et au Royaume-Uni,

41. OFCOM : Federal Office of Communications, OFGEM : Office of Gas and Electricity Markets, OFWAT : Water Services Regulation Authority.

42. ARCEP : Autorité de régulation des communications électroniques et des postes, CRE : Commission de régulation de l'énergie, ARAFER : Autorité de régulation des activités ferroviaires et routières.

43. Pour une analyse des motifs qui ont poussé le législateur français à créer un pouvoir de règlement dans ces domaines: Thomas Perroud, La fonction contentieuse des autorités de régulation en France et au Royaume-Uni, op. cit., p. 207-214. En 2014, le gouvernement a créé un Médiateur du livre, dont les pouvoirs sont proches du Médiateur du cinéma, mais il ne possède pas de pouvoir contraignant. 
rendraient impossible l'ouverture de ces secteurs à la concurrence (II.1). D'autre part, le droit privé des biens manifeste, en présence d'un réseau, de forts effets anticoncurrentiels (II.2).

\section{II.1. Le droit des contrats face aux secteurs régulés}

Quelles sont les raisons et la nature des agencements juridiques mis en place par le législateur ? Les contrats relatifs à l'accès et à l'interconnexion sont en effet, en France ${ }^{44}$, des contrats de droit privé - ce point est affirmé par le législateur dans certains domaines 45 - si bien que les autorités de régulation interviennent à la place du juge judiciaire lorsqu'elles règlent les différends. Au Royaume-Uni aussi, c'est le droit privé qui est concerné, il ne pourrait d'ailleurs en être autrement, car il n'existe pas de droit public des contrats.

Pourquoi le droit des obligations n'est-il pas adapté à la poursuite d'objectifs interventionnistes, à l'établissement d'un ordre concurrentiel ? Le règlement des différends devant les autorités de régulation offre une solution à différentes insuffisances du droit des contrats : ce droit, aussi bien en France qu'en Angleterre, n'offre pas de solution adaptée au problème du pouvoir dans les relations contractuelles, tout comme au refus de contracter et à l'établissement du contenu du contrat. Nous ne reviendrons pas sur l'indifférence du droit des contrats quant à l'inégalité des rapports contractuels car il est bien connu. C'est une critique ancienne en France et au Royaume-Uni, que l'on retrouve ainsi chez Max Weber, qui met en évidence que «l'égalité contractuelle formelle cache des inégalités réelles qu'elle contribue d'ailleurs à renforcer $» 46$. Nous nous concentrerons donc sur les deux autres points.

\section{Le droit des contrats et la décision de contracter}

Le problème du refus de contracter n'est pas traité par le droit des contrats anglais et français, si bien que ce droit apparaît mal adapté pour fournir une solution aux problèmes structurels des secteurs régulés.

Le droit civil des contrats repose sur le principe de liberté contractuelle lequel tient sa source dans la théorie de l'autonomie de la volonté ${ }^{47}$. Ce principe est commun aux deux pays étudiés ${ }^{48}$. Pour François Terré, Yves Lequette et Philippe Simler, la liberté contractuelle implique d'abord la liberté de contracter ou de ne

44. Cette distinction n'existe pas au Royaume-Uni; en tout cas, ce régime, comme régime distinct et homogène n'existe pas. Il y a en effet des régimes spéciaux encadrés par chaque loi, mais dans le silence de celle-ci, c'est le droit des contrats qui s'applique.

45. Voir, par exemple, l'article 34-8-I du Code des postes et des communications électroniques qui dispose que «l'interconnexion ou l'accès font l'objet d'une convention de droit privé entre les parties concernées ".

46. Jean-Guy BeLLEY, « Max Weber et la théorie du droit des contrats », Droit et Société, 9, 1988, p. 301-324, spéc. p. 302. La réflexion de M. Weber sur le droit des contrats se trouve dans : Max WEBER, Sociologie du droit, chapitre « Les formes de création des droits subjectifs », Paris : PUF, coll. « Recherches politiques », 1986.

47. Voir sur ce point, François TerRé, Yves LeQuette et Philippe SIMLER, Droit civil: les obligations, Paris : Dalloz, coll. "Précis Dalloz», 2009, p. 31. Voir aussi Laurent LeVENEuR, "La liberté contractuelle en droit privé : les notions de base (autonomie de la volonté, liberté contractuelle, capacité...) ", AJDA, 1998, p. 676.

48. Serge J. H. GiJRATH, Interconnection Regulation and Contract Law, Amsterdam : deLex B.V., 2006, spéc. p. 271 et suiv. 
pas contracter ${ }^{49}$. La jurisprudence en a tiré la conséquence que chacun doit être libre d'accepter de contracter sur le double fondement de la liberté du commerce et de l'industrie et du droit de propriété 50 .

Le common law refuse aussi de forcer un individu à contracter. Comme en droit français, il réglemente les raisons du refus qui ne peuvent être fondées sur un motif discriminatoire ${ }^{51}$ par exemple. Le common law connaît une exception à ce principe avec la jurisprudence relative aux common carrier $^{52}$. Les métiers que le juge ou le législateur qualifient ainsi sont dans une obligation de contracter. Les entreprises de services publics en réseau des secteurs étudiés sont ainsi toutes tenues de répondre aux demandes des usagers 53 . Une autre manière dont le common law traite le refus de contracter est l'angle du "restraint of trade». Si ce refus constitue une restriction déraisonnable au droit de toute personne d'exercer sa profession, celleci peut obtenir réparation devant les tribunaux ${ }^{54}$. Mais, pour Jack Beatson, aucune de ces deux voies n'a connu de développement au Royaume-Uni ${ }^{55}$. En l'absence de disposition législative précise, les tribunaux n'imposent pas d'obligation de contracter en droit anglais 56 . Faute d'une telle obligation, le droit des contrats semble bien inadapté pour répondre aux impératifs des secteurs régulés.

Ces principes du droit des contrats tant français qu'anglais apparaissent en contradiction frontale avec le régime des secteurs étudiés. L'objet de la fonction de règlement des différends est justement de surmonter le refus de contracter : les textes précisent bien que les différentes autorités peuvent être saisies dans des cas de " refus" ou de "désaccord" concernant l'accès ou l'interconnexion ${ }^{57}$. Il s'agit de réduire les prérogatives contractuelles de l'opérateur économique, ce que le juge français reconnaît d'ailleurs en affirmant « que, s'agissant de ses prérogatives contractuelles, les restrictions qui y sont apportées ont été voulues par le législateur qui,

49. François TerRé, Yves LeQuette et Philippe SIMLER, Droit civil : les obligations, op. cit., $\mathrm{n}^{\circ} 24$.

50. Ibib., $\mathrm{n}^{\circ} 125$.

51. Hugh Beale (ed.), Chitty on Contracts, Londres : Sweet \& Maxwell, $32^{\mathrm{e}}$ éd., 2015, $\mathrm{n}^{\circ} 1-016$. Il est ainsi interdit de discriminer une personne en raison de son sexe (voir Sex Discrimination Act, 1975, art. 6 [1]), de son origine raciale (Race Relations Act, 1976 art. 4 [1], 17, 20 et 21) ou de son handicap (Disability Discrimination Act, 2005).

52. La définition du common carrier donnée par le juge Bailhache dans la décision Belfast Ropework Co Ltd v Bushell est la suivante : "To make a man a common carrier he must carry as a public employment; he must carry for all indifferently; he must hold himself out as ready to carry for hire as a business and not as a casual occupation pro hac vice [i.e. on this occasion]. He is sometimes described as a person who undertakes for reward to carry the goods of such as choose to employ him from place to place. To this I think it would be safe to add the words "at a reasonable rate". All other carriers by land are private carriers", [1918] 1 K.B. 210.

53. Voir par exemple Gas Act, 1986, article 10 ; Electricity Act, 1989, article 16 (amendé par Utilities Act, 2000, article 44).

54. Jack BEATSON, "The Role of Statute in the Development of Common Law Doctrine», Law Quarterly Review, 2001, p. 258.

55. Ibid.

56. R v Disciplinary Committee of the Jockey Club, ex p. Aga Khan (1993) 1 W.L.R. 909.

57. Ce critère se retrouve dans les directives (voir par exemple l'article $12 \$ 1$ de la directive 2002/19/CE) et dans la loi (article L. 36-8 du Code des postes et des communications électroniques; article 38 de la loi du 10 février 2000 relative à la modernisation et au développement du service public de l'électricité). Dans le domaine du cinéma, on ne retrouve pas ces termes, mais ils sont implicites, car seul un refus peut justifier la saisine du Médiateur. La même remarque est valable dans le secteur audiovisuel. 
pour des motifs d'ordre public économique, a confié à l'Autorité de régulation, dans l'exercice de prérogatives de puissance publique, la mission d'imposer aux parties qui la saisissent, des décisions exécutoires tranchant leurs litiges sur la conclusion ou l'exécution d'une convention d'interconnexion ou d'accès à un réseau de télécommunication $" 58$.

Il n'y a pas d'arrêt au Royaume-Uni qui exprime la même idée dans un contexte identique : les décisions de règlement des différends ne sont pas mises en question sur ce fondement, si bien que le juge n'a pas eu l'occasion de se prononcer sur ce point. On peut toutefois établir une analogie avec des décisions juridictionnelles où le juge est venu accepter la restriction à la liberté contractuelle sur le fondement du droit de la concurrence ou du droit sectoriel. Le Competition Appeal Tribunal a ainsi exprimé cette idée, estimant que «la responsabilité des firmes dominantes l'emporte sur leur liberté de se comporter comme elles le souhaitent au détriment de la concurrence » 59 . De même, à l'occasion d'un recours en judicial review contre une injonction de l'Independent Television Commission, les juges ont estimé que l'autorité en question avait bien reçu du législateur la compétence de restreindre la liberté contractuelle des titulaires de licences 60 .

Cette analyse montre bien que le droit civil n'offre pas de solutions efficaces pour forcer à contracter afin d'ouvrir un secteur à la concurrence. C'est la raison, toute pragmatique, qui explique le recours à la procédure contraignante du règlement des différends devant l'autorité sectorielle. L'expérience, bien résumée par un manuel édité par la Banque Mondiale, montre en effet que si les concurrents sont laissés libres de négocier et, si les autorités en question refusent de trancher les litiges, on constate un retard dans l'ouverture du marché 61 .

La fonction de règlement des différends permet donc aux nouveaux entrants sur les différents marchés de trouver une solution tant à la question du pouvoir dans les relations contractuelles qu'à celle du refus de contracter des opérateurs dominants, lesquels n'y ont aucun intérêt. On verra ensuite que le règlement des différends s'affirmant nettement comme un mode de réglementation des contrats, il se distingue en cela du droit des contrats.

\section{Le droit des contrats et le contenu du contrat}

Par rapport au droit des contrats, le règlement des différends est bien un mode de réglementation. Cette fonction permet ainsi de conformer le contenu du contrat à l'intérêt général et de résoudre le problème de la fixation du prix dans les contrats.

L'office des autorités administratives de règlement des différends est de contraindre les relations contractuelles à respecter les principes posés par le législateur, à orienter les contrats dans une direction particulière. Ainsi, la doctrine en France n'hésite pas à affirmer que le règlement des différends est en réalité une forme de réglemen-

58. Cour d'appel de Paris, $1^{\text {re }}$ ch. H, 28 avril 1998, n 97/17847, France Télécom c. Sté Paris TV Câble (SLEC).

59. Office of Fair Trading v W. Austin \& Sons \& Ors [2005] CAT 25 (6 July 2005), spéc. § 337.

60. R. v Independent Television Commission Ex p. Flextech Plc [1999] E.M.L.R. 880.

61. Telecommunications Regulation Handbook, ed. par H. Intven, The World Bank, 2000, p. 1.21 
tation 62 , affirmation que l'on retrouve aussi au Royaume-Uni 63 et que les juges n'hésitent pas à rappeler 64 . En d'autres termes, il s'agit de contraindre les contrats à respecter les objectifs généraux de politique publique fixés par les textes. Ceci est très manifeste dans l'audiovisuel en France où les décisions de règlement des différends ont pour objectif de faire respecter l'objectif de pluralisme que le législateur et la Constitution requièrent du secteur 65 .

Ensuite, le droit sectoriel tente de résoudre le problème de la fixation du prix du contrat. On analysera d'abord séparément le problème de la fixation du prix en droit français et anglais. En droit français, le droit de la régulation tente bien de résoudre le problème de la fixation du prix, car «le prix est substantiellement un objet dont le droit ne se mêle pas » 66 . Comme le souligne Christophe Jamin, le droit sectoriel est dérogatoire en ce que « le principe de liberté des prix est très largement remis en cause ${ }^{67}$, ce que la cour d'appel de Paris a admis 68 . De même, MarieAnne Frison-Roche établit bien le caractère dérogatoire par rapport au droit commun des pouvoirs des autorités sectorielles :

Lorsque le contrat a pour objet l'accès au réseau, parce que nous sommes au cœur du droit de la régulation, la rencontre des volontés n'est plus la bonne référence. Il semble admis que le prix soit de fait unilatéralement fixé par le gestionnaire de réseaux, mais, contrepartie de ce pouvoir, il doit se justifier en toute chose et la preuve de l'adéquation entre le prix et la prestation repose sur lui, puisque toute somme correspondant à une prestation non justifiée en elle-même ou non confrontée à d'autres prestations qui auraient pu être moins onéreuses sera alors soustraite de la facture par le régulateur de la facture. À travers le règlement des différends, il y a donc bien une régulation des prix d'accès au réseau, aussi ferme qu'un mécanisme de tarification, et exercée comme telle par le régulateur. Le prix sera acceptable s'il est équitable au regard des coûts du raccordement même, mais aussi au regard des intérêts du cocontractant qui a une sorte de droit à la solution démontrée comme étant la plus économique. Ainsi, véhiculé par le principe procédural de transparence, se constitue un droit d'accès au prix «apparemment» le plus bas, au sens que la procédure donne à l'apparence,

62. Marie-Anne FrISON-Roche, «Régulation et règlement des différends : présentation du thème et synthèse du $10^{\mathrm{e}}$ forum de la régulation", Les Petites Affiches, 212, 22 octobre 2004, p. 6 ; ID., "Le pouvoir du régulateur de régler les différends. Entre office de régulation et office juridictionnel civil », p. 274 et suiv., in ID. (dir.), Droit et économie de la régulation. 3, Les risques de régulation, Paris: Presses de Sciences Po, Dalloz, coll. « Thèmes et commentaires », 2005.

63. OFCOM, Draft Enforcement Guidelines. Ofcom's Draft Guidelines for the Handling of Competition Complaints, and Complaints and Disputes Concerning Regulatory Rules, 6 July 2006, p. 31, §6.1.

64. Competition ApPeAl Tribunal, Hutchison 3G (UK) Limited v. The Office of Communications, 29 November 2005, [2005] CAT 39, § 138.

65. Voir, par exemple, la décision du Conseil supérieur de l'audiovisuel, décision n²007-471 du 17 juillet 2007 relative à un différend opposant les sociétés Voyage et CanalSatellite.

66. Marie-Anne Frison-Roche, «Qu'est-ce qu'un prix en droit ? Du droit des contrats au droit de la régulation", in Études à la mémoire de Fernand Charles Jeantet, Paris : LexisNexis, coll. « Mélanges », 2010, p. 177. Voir aussi, dans le même ouvrage, Christian MONTET, «Le droit de la concurrence : une nouvelle forme de dirigisme des prix? », p. 366 et suiv.

67. Christophe JAMIN, "Théorie générale du contrat et droit des secteurs régulés », Recueil Dalloz, 2005, p. 2342.

68. COUR D’APPEL DE PARIS, 20 janvier 2004, CT0175, S.A. Completel c. S.A. France Télécom. 
c'est-à-dire d'un prix qui se donne à voir comme étant le plus bas possible, et c'est sur le gestionnaire que repose l'obligation de prouver cette adéquation 69 .

Or, en droit civil français, le contrôle du prix dans le contrat n'est appréhendé par le juge que par le biais de l'abus 70 . Mais, ici encore, la sanction de cet abus est l'indemnisation ou encore la résiliation du contrat. «En aucun cas, la Cour de cassation n'autorise donc le juge à refaire le prix, si ce n'est de manière indirecte par l'octroi de dommages-intérêts, pas plus qu'elle n'exige de la partie, qui a déterminé unilatéralement le prix, qu'elle prouve que celui-ci n'est pas abusif 71 . " Il est possible, en suivant Muriel Chagny, de raffiner l'analyse en montrant que le droit commun des contrats ne se «soucie pas" des prix hauts et peu des prix bas 72 , problèmes plus adéquatement appréhendés par le droit de la concurrence ${ }^{73}$. Alors que le Moyen Âge s'est constamment préoccupé du problème du juste prix ${ }^{74}$, on ne peut que conclure : «le prix n'est guère apprécié dans son montant par le droit » 75 .

En droit anglais, le juge ne rentre pas non plus dans la question du prix. Ainsi les tribunaux anglais refusent-ils d'écarter les effets obligatoires du contrat «au motif qu'ils seraient inéquitables ou inégaux, par exemple lorsqu'un prix inadéquat a été consenti pour la vente d'une propriété »76. Néanmoins, c'est par le moyen de la notion de frustration 77 que le droit anglais des contrats a pu intégrer l'idée qu'un changement de circonstances pouvait rendre l'exécution du contrat commercialement impossible. Mais la sanction de la frustration, la fin rétroactive du contrat 78 , s'avère inadéquate aux secteurs étudiés puisque le but est bien d'assurer la poursuite de la relation. Il faut aussi ajouter que, dans le cadre de contrats spéciaux, le juge a pu imposer que le prix ait un caractère raisonnable ${ }^{79}$.

69. Marie-Anne FRISON-Roche, «Le droit d'accès au réseau de transport d'électricité pour un prix équitable (CA Paris, 1re ch., sect. H, 8 juin 2004, Cogé de Kerverzet) », Revue Lamy de la Concurrence, 1, 2004, spéc. p. 135.

70. Voir le commentaire de la jurisprudence de l'Assemblée plénière de la Cour de cassation du $1^{\text {er }}$ décembre 1995 par Christophe JAMIN, «Détermination unilatérale du prix: autoriser la résiliation du contrat cadre sans exiger la preuve d'un abus », in ID. (dir.), Droit et économie des contrats, Paris : LGDJ, coll. «Droit, économie », 2008, p. 93-113.

71. ID., "Théorie générale du contrat et droit des secteurs régulés », Recueil Dalloz, 2005 p. 2342.

72. Le droit des obligations peut appréhender ce problème par l'intermédiaire de la déloyauté. Le législateur est aussi intervenu pour prohiber la revente à perte (article L. 442-2 du Code de commerce) et les prix abusivement bas (voir article L. 420-5 du Code de commerce).

73. Murielle CHAGNY, Droit de la concurrence et droit commun des obligations, Paris : Dalloz, coll. « Nouvelle Bibliothèque de Thèses ", 32, 2004, p. 332, n 325.

74. Patrick Selim ATryah, The Rise and Fall of Freedom of Contract, Oxford : Clarendon Press, 1979, spéc. p. 61.

75. Marie-Anne Frison-Roche, «Qu'est-ce qu'un prix en droit ? Du droit des contrats au droit de la régulation », Regulatory Law Review, Les Petites Affiches, ${ }^{\circ}$ spécial, 6 mai 2010, n 90, p. 13 et suiv.

76. Hugh BEALE (ed.), Chitty on Contracts, Londres : Sweet \& Maxwell, op. cit., n 1-020.

77. Voir sur ce point, ibid., $\mathrm{n}^{\circ}$ 23-001 qui définit ainsi la frustration: « A contract may be discharged on the ground of frustration when something occurs after the formation of the contract which renders it physically or commercially impossible to fulfil the contract or transforms the obligation to perform into a radically different obligation from that undertaken at the moment of entry into the contract. "

78. La rétroactivité de l'annulation résulte d'une intervention législative (Law Reform [Frustrated Contracts] Act, 1943), auparavant la solution consacrée était la non-rétroactivité. Voir la position du common law: Hugh BeAle (ed.), Chitty on Contracts, op. cit., $\mathrm{n}^{\circ}$ 23-071.

79. Marie-Hélène MALEVILLE-COSTEDOAT, «Le contrat en Europe aujourd'hui et demain », Revue trimestrielle de droit civil (RTD Civ.), 2008, p. 741. 
Le droit privé des contrats en France et au Royaume-Uni n'apporte pas de solution satisfaisante aux problèmes que posent les secteurs de régulations, en raison de la puissance économique de l'opérateur dominant. Mais cette puissance est assise sur la propriété d'un réseau. C’est donc bien au fond les principes du droit privé des biens qui permettent d'expliquer l'attribution d'une fonction de règlement des différends aux autorités sectorielles.

\section{II.2. Le droit privé des biens face aux secteurs régulés : la notion d'accès}

Le caractère anticoncurrentiel du droit des biens a fait l'objet de peu d'études aussi bien en France qu'au Royaume-Uni. Ce n'est qu'en matière de propriété intellectuelle que l'attention des juristes sur cette question s'est focalisée. Dans ce domaine les études abondent, tant en langue anglaise que française, car la jurisprudence existe ${ }^{80}$. Pourtant, de nombreuses affaires en droit de la concurrence, de même que le régime juridique des secteurs régulés, montrent qu'il constitue la pierre d'achoppement de l'ouverture à la concurrence. La justification ultime du règlement des différends est peut-être même ici. Il permet à l'opérateur historique de conserver la propriété de son réseau tout en le forçant à l'ouvrir pour autoriser l'activité des concurrents.

On constate d'ailleurs que l'ouverture à la concurrence des industries en réseau a entraîné la mise en place, dans certains pays, de structures de propriété originales. Il fallait en fait séparer la propriété du réseau des activités opérationnelles des anciens opérateurs verticalement intégrés. Aux États-Unis, le modèle de «collaborative governance", mis en place dans le secteur de l'électricité, promeut une gestion du réseau par l'ensemble des parties prenantes, dans une structure, l'«opérateur indépendant de système », qui n'est pas censée faire des profits 81 . La mise en place de cette structure s'explique par la nécessité de séparer propriété du réseau et activités commerciales afin que l'opérateur de réseau n'ait pas d'incitation à fermer le marché. De même, au Royaume-Uni, le système choisi fut de créer une entité séparée possédant le réseau, la National Grid Company, qui n’a pas le droit d'investir dans la production ou la vente d'électricité 82 .

De même, les luttes entre les opérateurs historiques d'une part, et la Commission ou les autorités nationales de concurrence d'autre part, ont eu souvent pour objet la propriété du réseau ${ }^{83}$. La problématique est la suivante : comment mettre

80. Reto M. HiLty, «Individual, Multiple and Collective Ownership. What Impact On Competition? », Max Planck Institute for Intellectual Property and Competition Law Research Paper, $n^{\circ}$ 11-04, 2011.

81. Charles H. КосH Jr, «Collaborative Governance: Lessons for Europe from U.S. Electricity Restructuring», in Herwig C. H. Hofmann et Russell L. WeAver (eds.), Transatlantic Perspectives on Administrative Law, Bruxelles : Bruylant, coll. « Droit administratif/Administrative law », 7, 2011.

82. Michael G. PoLLIT, Lessons from the History of Independent System Operators in the Energy Sector, with applications to the Water Sector, EPRG Working Paper 1125, Cambridge Working Paper in Economics 1153, 2011.

83. Voir, par exemple, dans le domaine ferroviaire l'avis de l'Autorité de la concurrence française qui recommande une séparation juridique stricte et non pas seulement fonctionnelle entre la propriété des gares et des connexions et l'activité opérationnelle de la SNCF (Avis n 11 - A-15 du 29 septembre 2011 sur un projet de décret relatif aux gares de voyageurs et autres infrastructures de services du réseau ferroviaire) : Michel BAZEX, «Accès des concurrents de la SNCF aux gares de voyageurs ", Droit administratif, 12, 
en place une structure de réseau qui aurait intérêt à l'ouverture à la concurrence ? C'est bien une certaine conception du droit de propriété qui a entraîné, très certainement, l'éviction de la concurrence dans le domaine des réseaux. La notion d'accès, pourtant présente dans certains débats au XIX et au Xx siècle, fut écartée en raison précisément de la conception juridique de la propriété qui prévalait alors.

L'accès est aujourd'hui une notion cardinale du droit des biens dans les relations commerciales. Il est possible de le définir en utilisant trois critères. Le premier est relatif à sa nature : il s'agit d'un droit d'usage ou d'utilisation. C'est le seul critère stable, car les deux autres sont finalement des critères téléologiques. Ensuite, cet accès, matériellement, est relatif à certains biens essentiels. Enfin, fonctionnellement, il sert à l'exercice de certaines activités ${ }^{84}$. Il s'agit donc bien de forcer le détenteur d'une infrastructure essentielle à autoriser l'utilisation de son bien.

En conclusion, on peut donc dire que l'attribution d'une fonction de règlement
des différends s'explique, historiquement, au Royaume-Uni, par l'évolution de la
pensée économique et la volonté, en France, de promouvoir une certaine équité
dans des domaines importants. Si l'on essaye à présent de s'attacher aux raisons
structurelles qui ont pu expliquer l'émergence de ce pouvoir, c'est dans les carac-
tères du droit privé des contrats et des biens qu'il faut rechercher. L'étude de la
fonction de règlement des différends montre bien le changement de l'intervention
de l'État: il ne s'agit plus, comme dans le cadre de la police économique classique,
d'intervenir sur les prix ou l'intensité de la concurrence (par un système
d'autorisation). Il s'agit de promouvoir le degré de concurrence optimal, c'est-à-
dire le niveau de concurrence où le prix sera le plus faible tout en garantissant un
niveau satisfaisant de service au public. Telle est bien la mission de ce qu'il est dé-
sormais convenu d'appeler la régulation.

2011, comm. 103 ; Loïc GRARD, «La préparation des gares à l'ouverture du transport ferroviaire à la concurrence ", Revue de droit des transports, 11, 2011, comm. 169.

84. Thomas Perroud, La fonction contentieuse des autorités de régulation en France et au Royaume-Uni, op. cit., p. 570. 
- L'auteur

Thomas Perroud est professeur de droit public à l'Université d'Aix-Marseille, membre de l'Institut Louis Favoreu et futur professeur à l'Université Panthéon Assas - Paris 2. Ses recherches portent sur le droit public économique, le droit comparé et l'élaboration des politiques publiques. Il participe actuellement au Dictionnaire des communs (dirigé par Marie Cornu, Fabienne Orsi et Judith Rochfeld) et a récemment publié :

- Droit comparé de la procédure administrative (dir., avec Jean-Bernard Auby), Bruxelles : Bruylant, 2016;

- Droit et marché (dir., avec Sarah DORMONT), Paris : LGDJ, coll. «Droit \& Économie», 2015. 